\title{
Un horizon lourd d'inquiétudes... mais aussi de bonnes nouvelles
}

Dans une lettre adressée au secrétariat d'État à I'Enseignement supérieur et à la Recherche, le Conseil d'Administration (CA) de la Société Française de Physique (SFP), réuni en session le 26 juin 2014, a fait connaître son inquiétude induite par la politique nationale d'emploi scientifique, devenue trop timorée et inadaptée aux besoins de notre pays. Recherche et enseignement supérieur de qualité sont indispensables à la France, dans le contexte économique et culturel européen et international. La SFP est convaincue que I'emploi scientifique est I'investissement d'avenir par excellence. Il est vital d'envoyer un signal fort aux jeunes qui entrent aujourd'hui dans l'enseignement supérieur, en leur donnant l'envie et les moyens de faire de la recherche. Cet effort se double obligatoirement d'une politique efficace d'incitation à l'emploi des docteurs dans le secteur privé, notamment industriel, pour y développer durablement une culture de l'innovation, seule capable de restaurer une activité compétitive.

2014, Année Internationale de la Cristallographie, a stimulé et amplifié les liens naturels entre l'Association Française de Cristallographie et la Société Française de Physique. Plusieurs évènements marquants, visant publics scientifiques et publics non spécialistes, tout particulièrement des élèves de lycées et collèges, préparés avec beaucoup de compétence et de dévouement, ont touché un grand nombre de personnes. Ils ont permis de souligner que la science moderne, et bien sûr ses disciplines, la physique, la chimie, les géosciences, et plus récemment la biologie, se nourrissent de la cristallographie. Toujours émerveillé par la beauté des cristaux, le public découvre la richesse et la subtilité des applications dans la vie de tous les jours de ces objets aux symétries si séduisantes : ordinateur, téléphone portable, laser, capteurs...

La SFP et la Société Chimique de France publieront chacune dans leurs revues respectives, Reflets de la physique et L'actualité chimique, des numéros spéciaux complémentaires consacrés à la cristallographie. Après avoir abordé la question "Qu'est-ce qu'un cristal ? », le dossier " cristallographie » de Reflets, qui paraîtra en fin d'année, portera sur les développements des méthodes et des techniques les plus récentes de la cristallographie.

Ce dossier sera précédé en octobre par un numéro spécial «Femmes et physique », coordonné par la commission F\&P de la Société Française de Physique.

Ces 16, 17 et 18 juin 2014, I'Institut de Physique du CNRS, la Société Française d'Optique et la SFP avaient organisé à Agay les quatrièmes Journées des entrants dans les laboratoires de I'Institut de physique (JEPHY), pour contribuer à leur accueil au sein de la communauté physicienne et leur adresser un message de bienvenue au sein de la recherche, des laboratoires, de la profession et de ses acteurs... Les difficultés de transport n'ont pas permis cette rencontre. Ce n'est que partie remise. Elle se tiendra le $1^{\mathrm{er}}$ décembre, à Paris. Il y sera abordé, entre autres, la question des avantages d'une adhésion à la SFP : clairement, le profit le plus manifeste retiré de cette adhésion se trouve dans le dynamisme apporté à l'espace collectif professionnel que nous formons et développons chaque jour. Ne minimisons pas cependant l'apport fourni à l'individu par son appartenance à un réseau, élément essentiel d'une insertion professionnelle.

En 2014, pour la première fois, la SFP sera partenaire du «Prix La Recherche » pour la remise de ses grands prix. La cérémonie pour honorer les lauréats du Prix Jean Ricard et du « Prix spécial » se déroulera le mardi 21 octobre 2014, à 19 h, au musée du quai Branly. L'originalité de cette manifestation, réalisée en partenariat avec le journal La Recherche, tient à une présentation dense, à un auditoire important et à une forte couverture médiatique.

Clôturant la session du CA du 26 juin, nous nous sommes retrouvés, actuels administrateurs et beaucoup d'illustres prédécesseurs, pour accompagner Véronique Lemaître - agent général, mise à disposition de la SFP par le CNRS - dans son départ vers d'autres activités que lui ouvre une retraite bien méritée. Qu'elle trouve, dans ces quelques lignes et la chaleur du pot organisé le 26 juin, l'expression de notre profonde gratitude et des remerciements des divisions, des sections, des CA et des Bureaux de la SFP qui ont, tout au long de ces années, eu le privilège de bénéficier de son dynamisme au service des physiciens.

Alain Fontaine, Michel Lannoo Jean-Jacques Benattar et Charles de Novion 